\title{
Confinement effects in antiferromagnets
}

\author{
A. Díaz-Ortiz and J. M. Sanchez \\ Texas Materials Institute, The University of Texas at Austin, Austin, TX 78712
}

(Dated: October 24, 2018)

\begin{abstract}
Phase equilibrium in confined Ising antiferromagnets was studied as a function of the coupling $(v)$ and a magnetic field $(h)$ at the surfaces, in the presence of an external field $H$. The ground state properties were calculated exactly for symmetric boundary conditions and nearest-neighbor interactions, and a full zero-temperature phase diagram in the plane $v$ - $h$ was obtained for films with symmetry-preserving surface orientations. The ground-state analysis was extended to the $H-T$ plane using a cluster-variation free energy. The study of the finite- $T$ properties (as a function of $v$ and $h$ ) reveals the close interdependence between the surface and finite-size effects and, together with the ground-state phase diagram, provides an integral picture of the confinement in anisotropic antiferromagnets with surfaces that preserve the symmetry of the order parameter.
\end{abstract}

PACS numbers: 75.70.-i; 68.35.Rh

\section{INTRODUCTION}

Confinement effects play an important role in the thermodynamics of several materials such as polymers, liquid crystals, and magnets. For example, capillary condensation stands as a well known example of how phase equilibrium is affected by the confluence of surface and finite-size effects. In particular, due to the wall-particle interaction, a fluid between two plates undergoes a gasliquigtransition at a lower pressure than it does in the bulk. 12 . 3 . These effects of confinement are due to the additional contributions to the thermodynamic potential of the solvation force (finite-size effect) and the wall-fluid interfacial tension (surface effect).

A more complicated physical situation arises in the case of thin films of polymer mixtures on selective substrates.6 An $A B$ polymer mixture which undergoes a phase separation below a bulk critical temperature $T_{c}$, develops, when cast into a thin film over a substrate, an interface between the two phases which runs parallel to the substrate. This interface appears provided there is a substrate affinity for one of the components - the confinement is established between the polymer-air and polymer-substrate boundaries.

A model fluid confined between two parallel walls that exert opposite surface fields, has been often considered in order to investigate the upderying fursics in

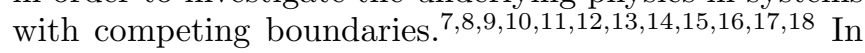
this case, the interplay between wetting and phase separation is very important, unlike the case of capillary condensation in which wetting plays a small role. The competition between surface effects leads to an interesting and unusual behavior: Phase coexistence is restricted to temperatures below the wetting temperature $T_{w}$ even in the limit of infinite separation between the plates. The wetting temperature depends on the surface field and it can be far from the bulk critical temperature 19 The aforementioned scenatip first predicted using a mean-field approximation, 8 has been confurmed subsequently via Monte Carlo simulations $11.12,13,44$ and transfer-matrix calculations in two dimensions. 15 However, when the effect of gravity is considered phase coexistence is restored up to the bulk critical temperature.16.17.18

The confinement studies described above deal with phase separating systems, in which the phases coexisting along a line of first-order transitions have the same symmetry, e.g., ferromagnetic thin films. Surface effects in systems with ordering (antiferromagnetic) interactions have been investigated mostly within the context of binary alloys undergoing a first-order phase transition, 20121.22 23.24 with particular emphasis on the surface-induced order and surface-induced disorder phenomena 25,26 although some investigations have been done in the context of multilayer adsorption.27,28,29 More recently, attention has turned to the surface critical behavior of bingry altars displaying continuous ordering reactions 24, $30.31,32.33,34.35$ and, in particular, to the dependence of the universality class on surface orientation. 3132

In this paper we investigate the interplay between finite-size and surface effects in Ising antiferromagnets in the presence of an external field. In particular, we are interested in systems with surfaces that preserve the symmetry of the order parameter. In other words, we shall study thin films which develop antiferromagnetic (AFM) ordering in each plane parallel to the surfaces. Our layered system can be described by the following Hamiltonian:

$$
\begin{aligned}
\mathcal{H}=J_{b} \sum_{i j \notin \text { surf }} \sigma_{i} \sigma_{j}+J_{s} \sum_{i j \in \text { surf }} \sigma_{i} \sigma_{j} \\
\quad-H \sum_{i \in \text { bulk }} \sigma_{i}-(h+H) \sum_{i \in \text { surf }} \sigma_{i},
\end{aligned}
$$

where the spin variable $\sigma_{i}$ takes the value of +1 or -1 depending if the spin at site $i$ is pointing up or down, respectively. We have assumed that surface sites, in layers 1 and $N$ for an $N$-layer film, experience a surface field $h$ in addition to the external magnetic field $H$. On physical grounds, it is natural to expect that the pair interactions at and near the surfaces differ from those 
TABLE I: Energies for the different ground-states discussed in the text. Nomeclature is as follows: structure $\uparrow / \uparrow \downarrow / \uparrow$ means that both surfaces are ferromagnetic (parallel to the applied field) and the remaining $N-2$ inner layers are antiferromagnetic. Structure $5^{\prime}$ is a special case in which the surface layers are ferromagnetic $(\uparrow)$, the subsurface layers are also ferromagnetic but in the opposite direction $(\downarrow)$ and the rest $(N-4)$ are antiferromagnetic $(\uparrow \downarrow)$. Bulk and surface coordination number are denoted by $z$ and $z_{s}$. See the text for further explanations.

\begin{tabular}{ccl}
\hline \hline Tag & Structure & \multicolumn{1}{c}{ Energy } \\
\hline 1 & $\downarrow / \downarrow / \downarrow$ & $\mathcal{H}_{1}=z_{s}+\left(\frac{1}{2} z+H\right)(N-2)+2(H+h)$ \\
2 & $\uparrow \downarrow / \downarrow / \uparrow \downarrow$ & $\mathcal{H}_{2}=-z_{s}+\left(\frac{1}{2} z+H\right)(N-2)$ \\
3 & $\uparrow / \downarrow / \uparrow$ & $\mathcal{H}_{3}=z_{s}-4 z_{1}+\left(\frac{1}{2} z+H\right)(N-2)-2(H+h)$ \\
4 & $\downarrow / \uparrow \downarrow / \downarrow$ & $\mathcal{H}_{4}=z_{s}-\frac{1}{2} z(N-2)+2(H+h)$ \\
5 & $\uparrow \downarrow / \uparrow \downarrow / \uparrow \downarrow$ & $\mathcal{H}_{5}=-z_{s}-\frac{1}{2} z(N-2)$ \\
$5^{\prime}$ & $\uparrow / \downarrow / \uparrow \downarrow / \downarrow / \uparrow$ & $\mathcal{H}_{5^{\prime}}=z_{s}+z_{0}-2 z_{1}-\frac{1}{2} z(N-4)-2 h$ \\
6 & $\uparrow / \uparrow \downarrow / \uparrow$ & $\mathcal{H}_{6}=z_{s}-\frac{1}{2} z(N-2)-2(H+h)$ \\
7 & $\downarrow / \uparrow / \downarrow$ & $\mathcal{H}_{7}=z_{s}-4 z_{1}+\left(\frac{1}{2} z-H\right)(N-2)+2(H+h)$ \\
8 & $\uparrow \downarrow / \uparrow / \uparrow \downarrow$ & $\mathcal{H}_{8}=-z_{s}+\left(\frac{1}{2} z-H\right)(N-2)$ \\
9 & $\uparrow / \uparrow / \uparrow$ & $\mathcal{H}_{9}=z_{s}+\left(\frac{1}{2} z-H\right)(N-2)-2(H+h)$ \\
\hline \hline
\end{tabular}

in the bulk. We approximate the position dependence of the pair couplings, by allowing the nearest-neighbor intralayer surface coupling $\left(J_{s}\right)$ to differ from the bulk one $\left(J_{b}\right)$. Here we restrict ourselves to case of $J_{b}>0$ (antiferromagnetic), but we allow $J_{s}$ to assume any real value. Also, we specialize ourselves in the case of localized symmetric surface fields, i.e., the field at each surface is the same and acts only at the surface sites. In the remaining of the paper, the effective pair interactions, the surface field, and the external magnetic field $(H)$ shall be expressed in terms of the bulk AFM coupling $\left(J_{b}>0\right)$. The ratio of surface to bulk coupling is then denoted by $v$.

Confinement effects in the order-disorder transitions for the particular case of $v=1$ and $h>0$ have been reported previously. 36 In this paper, we give a full description of the surface and finite-size effects in terms of the variables $h$ and $v$. The ground-state properties of the Hamiltonian (1) are derived in Sec. II. This zerotemperature analysis is used to identify the different sequences of ground states displayed by the film as a function of the external field $H$. Moreover, it is shown that for antiferromagnetic systems with symmetry-preserving surface orientations and nearest-neighbor interactions, a zero-temperature phase diagram can be drawn as a function of $v$ and $h$, for any value of the number of layers $N$ and external field $H$. In Sec. III, we use a cluster variation free energy 37 to describe the finite-temperature behavior of the system as a function of surface variables $v, h$ and the number of layers $N$. Particular attention is devoted to the analysis of the critical curve (in the $H-T$ plane) for each one of the different regions of the zerotemperature phase diagram. We close with a summary of the important results (Sec. IV).

\section{GROUND-STATE PROPERTIES}

In the absence of surface and finite-size contributions, that is in the bulk, the Hamiltonian (11) reduces to

$$
\mathcal{H}_{\text {bulk }}=\sum_{i j} \sigma_{i} \sigma_{j}-H \sum_{i} \sigma_{i}
$$

For a two-sublattice antiferromagnet such as bodycentered or simple cubic, the Hamiltonian (2) has three different ground states as a function of the external field $H$ : ferromagnetic $(\downarrow)$ for $H<-H_{c}$; antiferromagnetic $(\uparrow \downarrow)$ for $-H_{c}<H<H_{c}$ and again ferromagnetic ( $\uparrow$ ) for $H>H_{c}$. The critical field $H_{c}$, equal to the coordination number $z$ [recall that all quantities in Eq. (11) as well as in Eq. (2) are normalized to $J_{b}$, determines the point where the critical curve $T_{c}(H)$ meets the field axis.

For the AFM thin films studied here [see Hamiltonian (11)], the possible ground-state (GS) structures are listed in Table If along with their corresponding energy. We considered only the case $h>0$ since the results for $h<0$ can obtained straightforwardly from the symmetry properties of Hamiltonian (11). The nomenclature in Table fis as follows: structure number 4 corresponds to $\downarrow / \uparrow \downarrow / \downarrow$, which means that both surfaces are ferromagnetic $(\downarrow)$ and that the remaining $(N-2)$ inner layers are antiferromagnetically ordered. Structure $5^{\prime}$, a special case to be discussed later in the paper, has both surfaces in a ferromagnetic state $(\uparrow)$, the subsurface layers are ferromagnetic but with magnetization in the opposite direction $(\downarrow)$, and the remaining $(N-4)$ layers are antiferromagnetic.

We arrived at the set of GS in Table 1 as follows. Since only nearest-neighbor interactions are included in the Hamiltonian and the (uniform) surface field acts locally at surface sites, the presence of long-period superstructures can be ruled out. A possible set of ground states 

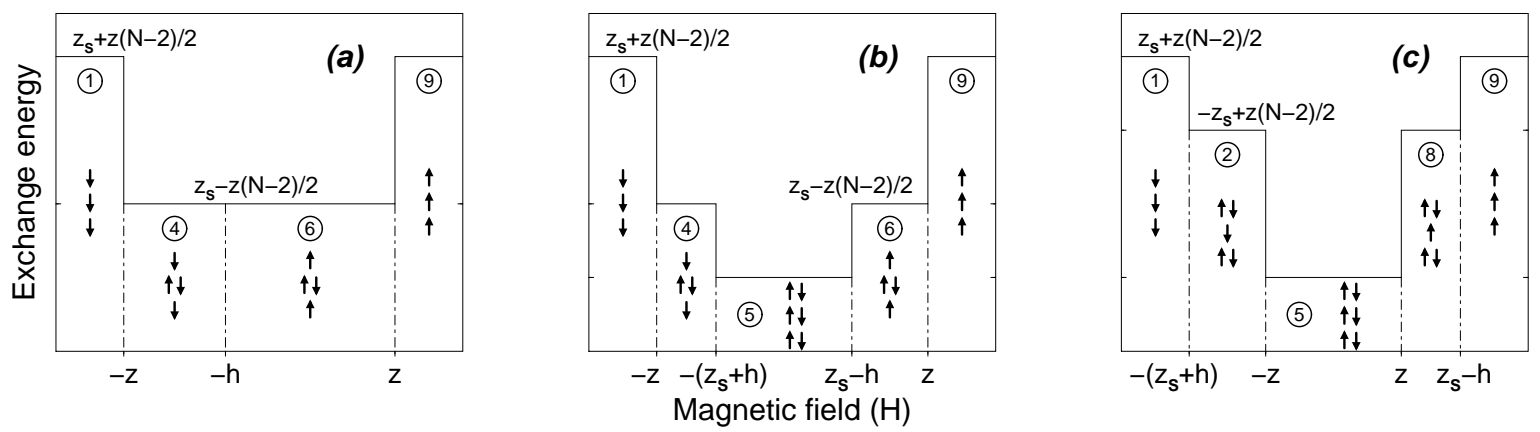

FIG. 1: Schematic ground-state sequences VII (a), I (b), and II (c). The succession (a) to (c) represents the evolution in the ground-state sequence as we increase the surface pair interactions for neutral boundary conditions. The domain of stability of each structure is different from each other, but for $h=0$ the transition I $\rightarrow$ VII occurs at $v=v_{p s}=-z_{1} / z_{0}$ for which GS 5 in (b) has shrunk to zero width. The range of stability of GS 5 expands as we increase $v$. At $v=v_{m}=\left(z_{0}+z_{1}\right) / z_{1}$ a transition between I in (b) and II in (c) occurs. As a reference, the value of the exchange energy for selected GS is indicated in the plots. See Fig. 2 for the evolution of sequences I and II with $h$. Also see the text for further explanations.

for Hamiltonian (11) was then constructed by combining all possible surface and bulk ground states. For the sake of definiteness, let us consider a body-centered cubic film with surfaces in the (110) direction. The bulk ground states consist of two ferromagnetic structures (with opposite magnetization) plus an ordered CsCl-type AFM structure. The (110) surfaces constitute face-centered rectangular lattices, for which the possible ground states are a checkerboard AFM structure and two ferromagnetic states of opposite magnetization. The nine ground-state structures obtained by combining the surface and bulk ground states are listed in Table 1 . These structures are ground states of Hamiltonian (11) in the limit of weak coupling between the surface and the subsurface layers. For strong coupling between the surfaces and the bulk, we found only-gne additional ground-state structure- GS 5' in Table 38

The Hamiltonian in Eq. (11) distinguishes between the pair interactions in the surface layers from the rest, thus allowing us to define the surface coordination number $z_{s}$ as a function of the surface coupling parameter $v$

$$
z_{s}(v)=z_{0} v+z_{1},
$$

where the intralayer and interlayer coordination are denoted by $z_{0}$ and $z_{1}$, respectively. Recall that all quantities in Eq. (11) are given in terms of $J_{b}$ and, therefore, $z_{s}$ in (3) actually accounts for the surface energy. For a $\operatorname{bcc}(110)$ film, $z_{0}=4$ and $z_{1}=2$, and the bulk coordination number is $z=z_{0}+2 z_{1}$.

Even in the absence of an applied surface field $h$, the surfaces are under the influence of a "missing neighbors" field $h_{m}(v)$ that arises from the disruption of the translational symmetry perpendicular to the surfaces. This missing neighbors field produces an inhomogeneous magnetization profile. Thus, with increasing external field, the surfaces may turn into a ferromagnetic state before the bulk does. Application of a surface field $h=-h_{m}(v)$ can restore the magnetization profile to the homogeneous condition. Note that the missing neighbors field depends on $v$, since $h_{m}$ is a measure of the difference between the environment at the surfaces and in the bulk [see Eq. (3)]. The missing neighbors field can be written as

$$
h_{m}=z_{s}-z=z_{0} v-\left(z_{0}+z_{1}\right) .
$$

We derive this value for the missing neighbors field later in paper, by considering the stability of the different GS structures as a function of $v$ and $h$.

A direct comparison between the energies $\mathcal{H}_{i}(v, h, H, N)$ for each structure $i$ gives the ground state for every set of values of the thermodynamic variables (see Table II). However, it is more useful and less tedious to consider a physical sequence of GS structures (as a function of the applied field) and examine its domain of stability as we vary the surface variables $v$ and $h$.

As a starting point, consider the following case: Upon the application of an external field $H$ (in either direction), a film with $v \sim 1$ and $h \sim 0$ will pass from an AFM state in all layers (small $|H|$ ) to a state with ferromagnetic surfaces and an AFM bulk and, finally, for large $|H|$, to a ferromagnetic state in all planes. This case is represented by the sequence 1-4-5-6-9 of GS structures [a schematic view is presented in Fig. 1 (b). See also Table for the nomenclature]. The characteristic value of the external field at the transition between different GS structures is indicated in Fig. 1. In general, the transition between GS structures $A$ and $B$ occurs at $\mathcal{H}_{A B}$, which is determined by equating the corresponding energies.

Ground-state sequence 1-4-5-6-9 (hereafter referred as I) in Fig. 1 (b), provides some useful insight on confinement versus finite-size effects. At the beginning of this Section we considered the ground states of an infinite antiferromagnet, which in the nomenclature of Table il, correspond to GS sequence 1-5-9 in the limit of $N \rightarrow \infty$. Thus, ground-state structures 4 and 6 are due the confinement effects. When either GS 4 or GS 6 become unstable in favor of GS 5, surface effects are lost and the film is subject only to the finite-size effects.

An external surface field will produce an asymmetry in the GS sequence since the Hamiltonian is not invariant 
under the transformation $\sigma_{i} \rightarrow-\sigma_{i}, H \rightarrow-H$. Applying a surface field $h>0$ reduces the surface ferromagnetism $(\downarrow)$ in GS 4 and enhances it in GS $6(\uparrow)$. The domain of stability of GS 4 shrinks to zero when $z_{s}+h$ becomes $z$. This particular value of $h$ defines the missing-neighbors field [Eq. (4)].

Applying an external surface field is not the only way to eliminate surface effects in AFM thin films. A homogeneous condition can also be attained in the film by setting neutral boundary conditions $(h=0)$ and increasing the pair interactions at the surfaces to a given value $v_{m}$. The characteristic value of the surface coupling that compensates for the missing neighbors effect is given by:

$$
v_{m}=\left(z_{0}+z_{1}\right) / z_{0} .
$$

For this value of the surface coupling GS 4 and GS 6 become unstable simultaneously [Eq. (5) is equivalent to the condition $\left.z_{s}=z\right]$.

For values of the surface coupling larger than $v_{m}$, keeping the neutrality at the boundaries, ordering becomes stronger at the surfaces than in the inner layers. This situation is represented in Fig. 11(c). It is worth noting that the GS sequence 1-2-5-8-9, hereafter referred as II, is stable not only in the case of $h=0$ but for a range of values of $v>v_{m}$ and $h$. We will return to this point later in the paper.

Reducing the surface coupling make surface ordering less stable, until $v$ reaches the characteristic value

$$
v_{p s}=-z_{1} / z_{0}
$$

for which GS 5 becomes unstable $\left[v_{p s}\right.$ in (6) corresponds to the condition $\left.z_{s}=0\right]$. The remaining ground-state sequence 1-4-6-9 (hereafter VII) are depicted in Fig. 11(a). Sequence VII remains unaltered for $v<v_{p s}$ regardless the strength of $v$ : The phase coexistence between spin-up and spin-down is regulated by the surface field $h$. Large, negative values of $v$ increase the critical-point temperature. In the alloy terminology, sequence VII represents the situation of a binary-alloy thin film with an ordered bulk coexisting with a surface miscibility gap. This will become apparent in Sec. III where we discuss the finitetemperature properties of Hamiltonian (11).

Sequences I, II, and VII (Fig. 11) were obtained by analyzing the stability of the corresponding GS sequences upon variations of the surface coupling $v$ for neutral boundary conditions. As expected, a similar variation of GS sequences will appear as we increase the surface field. Consider for example sequence II in Fig. 1(c): Setting higher values for the surface field eventually overcome the ordering tendencies at the surfaces. Ground-state structure 8 then becomes unstable and sequence II turns into the new 1-2-5-6-9 GS sequence (III) depicted in Fig. 2(a). The asymmetry of sequence III is interesting. For very negative values of $H$ sequence III looks like sequence II (in the same range of $H$ ), with long-range order dictated by the surfaces. On the other hand, for large positive values of $H$, sequence III looks like sequence I, for which
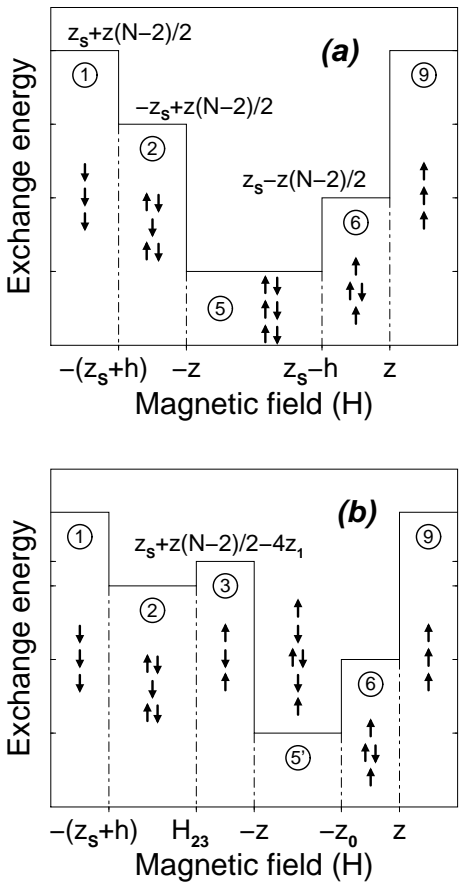

FIG. 2: Sequence II in Fig. 11(c) becomes sequence III in (a) as the surface field is increased. The transition II $\rightarrow$ III occurs at $h_{\mathrm{II}-\mathrm{III}}=z_{0} v-\left(z_{0}+z_{1}\right)$ when GS 8 becomes unstable (see Table III). A further increase of the surface field $h$ establishes 1-2-3-5'-6-9 in (b) as the stable GS sequence (IV). Observe the appearance of GS $5^{\prime}$ and the disordered gap between GS 3 and GS $5^{\prime}$. The characteristic field between GS 2 and GS 3 is $H_{23}=z_{s}-2 z_{1}-h$. See the text.

the bulk is responsible for the AFM ordering. This similarity is due to the fact that sequence I evolves into III when the surface field increases beyond $h=z-z_{s}(v)$ (missing neighbors field) for $0<v<v_{m}$.

The homogeneous antiferromagnetic thin film (GS 5), with constant energy for given $v$ and $N$, becomes rapidly unstable with increasing $h$. For sufficiently large $h$, GS 5 is replaced by another zero-magnetization structure, GS $5^{\prime}$ in Table 1 , with energy given by

$$
\mathcal{H}_{5^{\prime}}=z_{s}+z_{0}-2 z_{1}-\frac{1}{2} z(N-4)-2 h .
$$

For a given value of the number of layers $N$ and the coordination at the surfaces, $\mathcal{H}_{5}$ is constant while $\mathcal{H}_{5^{\prime}}$ depends only on $h$. When the surface field reaches the value of

$$
h_{\mathrm{III}-\mathrm{IV}}=z_{0} v+\left(z_{0}+z_{1}\right),
$$

structures 5 and $5^{\prime}$ have the same energy. A unique feature of GS 5 and GS $5^{\prime}$ is that they remain degenerate over a finite range of the external field $H$. From Eq. (8) and Fig. 2(a) we can see that the ground state for an AFM film at a surface field value given by (8) is a mixture of GS 5 and GS $5^{\prime}$ for $H \in\left(-z,-z_{0}\right)$. On average, a scan in $H$ will show a layer magnetization of $+\frac{1}{2}$ at the surfaces together with subsurface magnetization of $-\frac{1}{2}$ and AFM bulk (zero magnetization). Thermal excitations destroy this degeneracy between GS 5 and GS 5' 

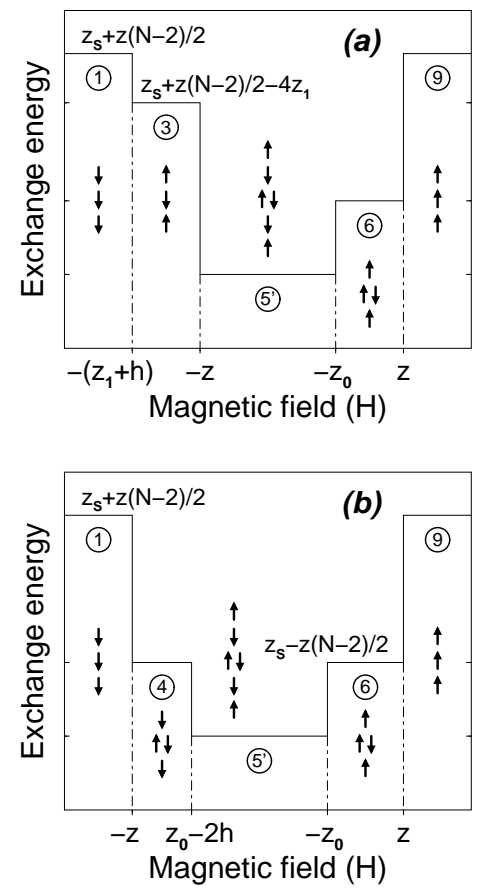

FIG. 3: Ground-state sequences for intense surface fields and negative $v$. Sequence $\mathrm{V}$ in (a) is obtained from $\mathrm{IV}$ in Fig. 2 (b), when the surface AFM-phase in GS 2 becomes unstable upon the reduction the pair interaction at surfaces. A coexistence between spin-up (GS 3) and spin-down (GS 1) magnetizations is established at the surfaces [cf. Fig. 1(a)]. For sufficient negative values of $v$ and large $h$, a line of firstorder transitions, ending at a critical point, occurs outside the antiferromagnetic critical curve. Sequence I in Fig. 1(c) turns into sequence VI in (b) in the same way as III becomes IV (Fig. 2), that is, replacing GS 5 by GS $5^{\prime}$. A difference arises, however, since in this case GS 4 is adjacent to GS $5^{\prime}$, and a coexistence line will appear inside the AFM region. See the text for details.

in most of the interval $\left(-z,-z_{0}\right)$ in favor of GS 5 , except near the ends, i.e. $H \sim-z$ and $H \sim-z_{0}$, where GS $5^{\prime}$ is pinned by the onset of stability of GS 3 and the presence of GS 6 . Traces of the ground-state degeneracy between GS 5 and GS $5^{\prime}$ are observable at low temperatures. For the other structures listed in Table [1, a transition similar to $5 \rightarrow 5^{\prime}$ does not occur, mainly due to the symmetry in the boundary conditions.

Ground-state sequence III evolves into 1-2-3-5'-6-9 sequence (IV hereafter) at $h=h_{\text {III-IV. The situation is }}$ shown schematically in Fig. 2(b) for $h>h_{\text {III-IV. Ob- }}$ serve that the appearance of GS 3 has established a disorder gap between GS 2 (AFM surfaces) and GS 5' (AFM bulk). This behavior is unique in the sense that in all previous cases the ordered domain was a compact interval in $H$. This characteristic brings some interesting features into the $H-T$ phase diagram, such as the splitting of the film's critical $T_{c}(H)$ curve into two distinct critical curves. 36

Increasing the surface field does not change sequence IV into another GS sequence. However, for a large value of $h$, antiferromagnetic order at the surfaces becomes unstable upon reduction of the surface coupling, and IV changes into sequence $\mathrm{V}$ composed of GS sequence 1-3$5^{\prime}-6-9$ for $v<0$ [Fig. 3(a)]. Observe in Fig. 33(a) that GS 1 is now adjacent to GS 3. The difference between GS 1 and GS 3 resides at the surfaces, which have opposite magnetization. This situation is reminiscent to the one found in sequence VII [Fig. II(a)] where the surface field regulates the surface phase coexistence between upand down-magnetizations. In sequence $\mathrm{V}$, however, the line of first-order transitions is located outside of the well defined AFM region composed by GS $5^{\prime}$ and GS 6 .

Ground-state sequence V is stable for large $h$ and negative $v$. Previously, we found that VII is the stable GS sequence for $v<v_{p s}$ and low $h$. A transition between $\mathrm{V}$ and VII certainly occurs, although it is mediated by the GS sequence 1-4-5'-6-9 (VI) [see Fig. 3(b)]. Finally, sequences I and VI are separated by the GS 5 to GS $5^{\prime}$ transition, which in this case occurs at lower values of $h$ since $v<0$.

We have discussed the several GS sequences that appear in confined antiferromagnets along particular paths, namely, we fixed $h=0$ and varied the surface coupling (Fig. 1) or alternatively, we fixed $v>v_{m}$ and increased $h$ (Fig. 2). In general, however, the transition from one GS sequence to another does not occur at constant $v$ or $h$. The relationship between the surface variables $(v, h)$ at the different transitions between GS sequences (see Table III), defines the domain of stability of each sequence, from I to VII, in the plane $v$-h. The corresponding ground-state phase diagram is shown in Fig. 4 . The phase diagram is symmetric with respect to $h=0$, with the negative- $h$ region obtained by replacing spin-up with spin-down and $H$ with $-H$ in Fig. 1. The zero-temperature phase diagram provides a good reference frame to interpret some of the features reported in previous work pinary-alloy thin films with ordering interactions. 36 39. 40.41 The ground-state phase diagram is also a valuable guide for the investigation of the finitetemperature properties of Hamiltonian (11) to be carried out in the next Section.

\section{FINITE-TEMPERATURE PROPERTIES}

The finite-temperature properties of Hamiltonian (1) were calculated using the cluster-variation method (CVM) in the pair approximation (PA). 10 For the twosublattice antiferromagnets considered in this paper, the physical aspects of phase equilibrium under confinement are well captured by the PA-CVM 3641 For bcc(110) films with neutral boundary conditions, a comparison between the PA and the tetrahedron approximation (TA) has shown that only the quantitative aspects are improved with the latter.11 For a general exposition of the clustervariation method, we refer the interested repder to the excellent reviews available in the literature 42.43,44,45.46

The order-disorder transitions are described in the 


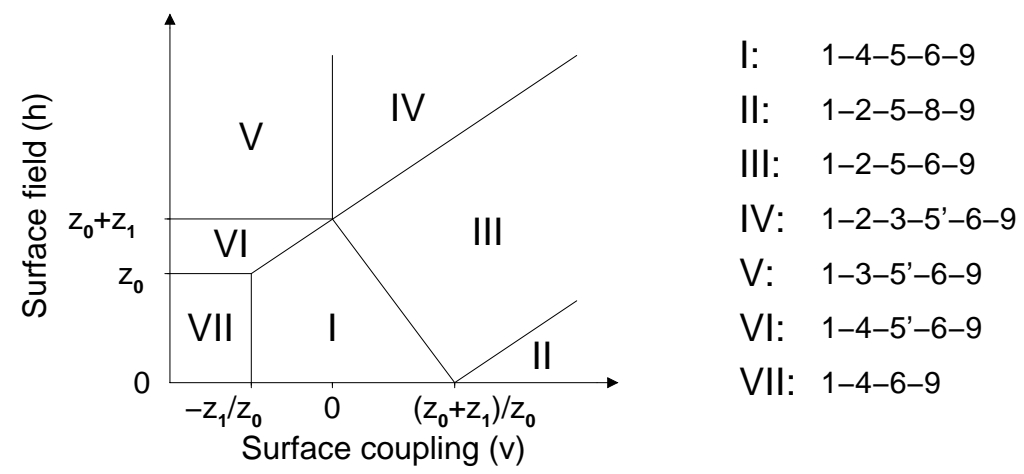

FIG. 4: Ground-state phase diagram $v$ - $h$ for antiferromagnetic thin films. Table II contains the characteristic values of the surface field that describe the boundary between the different regions. Regions I-III define compact antiferromagnetic domains, while for region IV a disordered (ferromagnetic) gap intervenes between the AFM order at the surfaces and the ordered bulk. For large negative values of the surface coupling, a line first-order transitions ending in a critical point, appears in regions V-VII. The intra- and interlayer coordination numbers are denoted by $z_{0}$ and $z_{1}$, respectively. See the text for further explanations.

usual manner by subdividing the bcc or sc lattice into two interpenetrating sublattices $\alpha$ and $\beta$. The long-range order parameter in the $k$-layer defined as

$$
\eta_{k}=\frac{1}{2}\left(m_{\alpha}^{k}-m_{\beta}^{k}\right)
$$

where $m_{\alpha(\beta)}^{k}$ is the $\alpha(\beta)$-sublattice magnetization in the $k$ layer.

TABLE II: Characteristic values of $h$ and $v$ defined by the transitions between different ground-state sequences. These characteristic values define the domain of stability of structures I to VII. See phase diagram in Fig. 1 and the text for additional details.

\begin{tabular}{ll}
\hline \hline \multicolumn{1}{c}{ Surface field $/$ coupling } \\
\hline$h_{\text {I-III }}=-h_{\mathrm{II}-\mathrm{III}}=-z_{0} v+\left(z_{0}+z_{1}\right)$ \\
$h_{\mathrm{I}-\mathrm{VI}}=h_{\mathrm{III}-\mathrm{IV}}=z_{0} v+\left(z_{0}+z_{1}\right)$ \\
$h_{\mathrm{V}-\mathrm{VI}}=z_{0}+z_{1}$ \\
$h_{\mathrm{VI}-\mathrm{VII}}=z_{0}$ \\
$v_{\mathrm{I}-\mathrm{II}}=\left(z_{0}+z_{1}\right) / z_{0}$ \\
$v_{\mathrm{I}-\mathrm{VII}}=-z_{1} / z_{0}$ \\
\hline \hline
\end{tabular}

With reference to the GS phase diagram of Fig. 母, regions I-III display long-range order, either at the surfaces (GS 2 and 8 ) or in the bulk (GS $4=6$ ). With the exception of sequence I, the critical curves 47 obtained in regions II and III show a distortion at high temperatures. Our results for the critical curve in these regions, summarized in Fig. 5 , can be explained using the ground-state analysis discussed in Sec. II.

Phase diagrams in region I are virtually independent of the parameters $v$ and $h$, as can be seen in Fig. 5 (a). This behavior can be attributed to the fact that the AFM ordering in region $I$ is primarily due to the inner layers [see Fig. 1.(b) and Fig. [1. Thermal excitations can promote spin flip at the surfaces, resulting in a lower degree of ordering at surfaces relative to the bulk. In contrast, region II is characterized by a strong AFM ordering at the surfaces coupled with the AFM bulk [see Fig. 11(c)], thus preventing the formation of a (separate) surface critical curve. Instead, the $H-T$ phase diagram shows an increase in the transition temperature and a broadening in the external field region for which the stable phase is antiferromagnetic. A relative small asymmetry in the critical curve is observed, due to the fact that the surface field favors the stability of GS 2 over GS 8 [Fig. 国(a)]. Thus, the distortion in the phase diagrams associated with region II stems from the relative stability of two ground-state configurations with the same symmetry, i.e., GS 2 and GS 8.

A higher asymmetry in the phase diagrams is expected in region III, since the critical-curve shape is dictated by the surface ordering for $H \sim-\left(z_{s}+h\right)$, and by an AFM bulk (with low surface ordering) for $H \sim z$. The difference in symmetry of the AFM structures at each AFM:FM boundaries [see the GS sequence in Fig. 2(a)], allows the surfaces to drive the phase transition for fields close to the (negative) critical field value. One can see that the surfaces are developing their own critical curve, which unfolds as a 'shoulder' in the phase diagram for negative applied field [see Fig. F(b)]. Characteristics such as the maximum temperature of the shoulder or its extension in $H$, are controlled by the surface variables $v$ and $h$. The critical field between GS 1 and GS $2\left[H_{12}=-\left(z_{v}+h\right)\right]$ makes apparent that the extension of the shoulder depends on the surface field. The maximum temperature in the shoulder is about $v T_{\text {surf }}$, where $T_{\text {surf }}$ is the Néel temperature of the corresponding surface antiferromagnet. Here, as in the rest of the paper, the relevant thermodynamic variables are expressed in units of the (positive) AFM coupling. Thus, in the PA a square lattice has a maximum critical temperature $k T_{\text {surf }}=4 / \ln 4 \approx 2.88$.

As pointed out previously, region IV is characterized by the formation of a disordered gap between two different ground states [see Fig. 2(b)]. At finite temperatures and deep inside region IV, the surfaces develop their own critical curve well separated from the bulk antiferromagnetic region [see Fig. 6(a) showing the critical curves for 

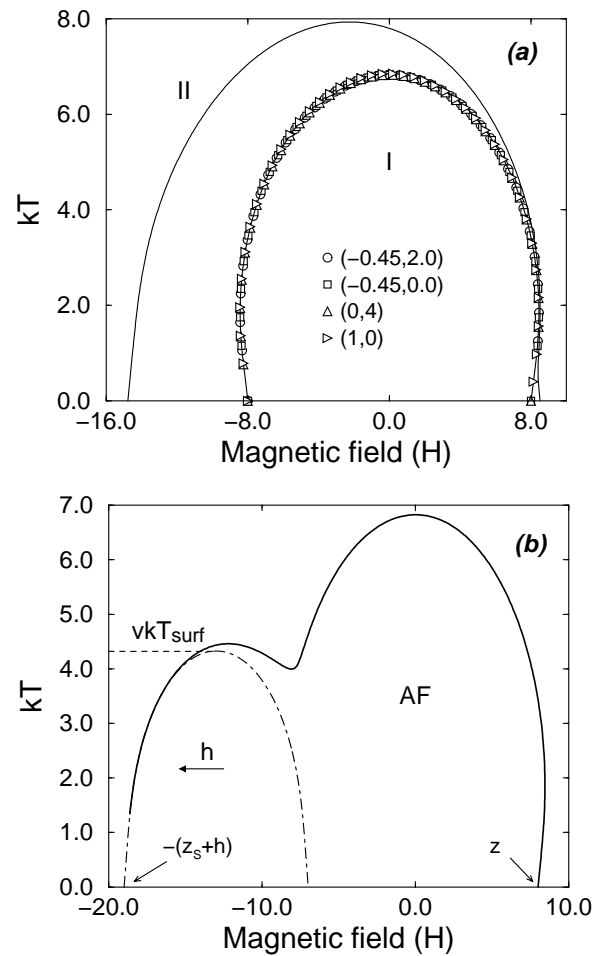

FIG. 5: (a) Typical critical curves for regions I (symbols) and II (solid line, $v=2.41$ and $h=3.14$ ). The various symbols correspond to different values of the surface variables $(v, h)$ as shown in the inset. Note that in region I the shape of the critical curve is virtually independent of $v$ and $h$. (b) Phase diagram for region III (solid line), showing the development of a 'shoulder' as a signature of the incipient surface critical curve. The values for the surface variables are $v=1.5$ and $h=11$. The antiferromagnetic domain is a compact region. The phase diagram for a square lattice is shown as reference (dot-dashed line) and to illustrate the process of separation between the bulk and surface critical curves ( $c f$. Fig. 6). Both in (a) and (b) antiferromagnetic bcc(110) films with $N=14$ were considered and solved in the pair approximation of CVM.

a 14-layer film with $v=1.5, h=14$ (circles) and $h=18$ (triangles)]. Since the surfaces are weakly coupled with the bulk, the surface critical curve scales with $v$, i.e., the zero-temperature width of the AFM ordering is $z_{0} v$ and the maximum critical temperature is $v k T_{\text {surf }}$.

Between the situation of unconnected ordered domains and the phase diagrams observed in region III, there is the case in which the zero-temperature disordered gap transforms, via thermal excitations, into a disordered region in the $H$-T plane right inside the compact $\mathrm{AFM}$ domain. An increment in the surface field translates into an increment in the height of the disordered region. At $h=h_{s}$ the AFM region splits into the surface and the bulk critical curves [see Fig. 6(b)]. At finite temperatures, the splitting value of the surface field $h_{s}$ plays the role of $h_{\text {III-IV }}$ : for $h<h_{s}$ the ordered region is compact whereas for $h>h_{s}$ there are two unconnected critical curves.

Expressing the free energy $F$ in terms of the long-range
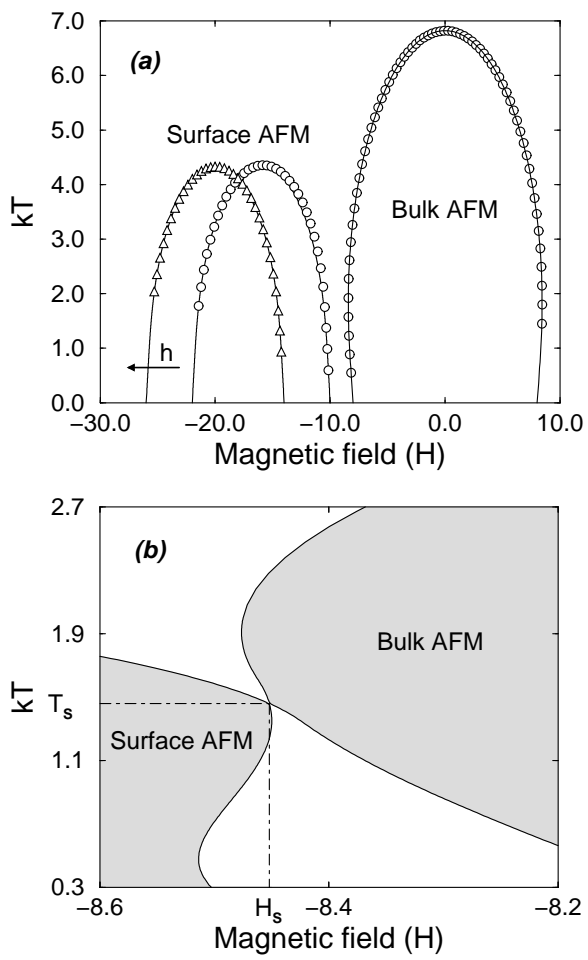

FIG. 6: For an intense field at the boundaries $\left(h>h_{s}\right)$, the surfaces decouple from the inner layers (bulk) and develop their own critical curve. In (a) the critical curves of 14-layer antiferromagnetic thin films are shown for $v=1.5, h=14$ (circles) and $v=1.5, h=18$ (triangles). The bulk critical curve showed no difference from $h=14$ to $h=18$, hence only the former case is depicted. The solid lines represent, in the case of the surface critical curves, the phase diagram of a square AFM, appropriately shifted. The solid line in the bulk phase is the one associated to $N=12$ in region I. The splitting between the surfaces and bulk critical curves occur at $T_{s}$ (temperature of splitting) and $H_{s}$ (field of splitting), when the surface field reaches the value of $h_{s}$. Part (b) shows a detail of the phase diagram of 100-layer AFM film at the very point of splitting.

order parameters (9), the conditions determining the locus of the splitting point are given by:

$$
\begin{gathered}
\lambda=\operatorname{det}\left(\frac{\partial^{2} F}{\partial \eta_{k} \partial \eta_{k^{\prime}}}\right)=0, \\
\frac{\partial \lambda}{\partial T}=0, \quad \frac{\partial \lambda}{\partial h}=0 .
\end{gathered}
$$

Equation(10a) defines the critical temperature, at fixed external conditions $(T, H, h$ and $N)$, when the second derivatives of the free energy are evaluated in the disordered state.48 Since $\lambda<0$ in the ordered state, at the splitting point $\left(T=T_{s}\right) \lambda$ is a concave function of the external field vanishing at the splitting value of the magnetic field $H_{s}$. In a similar fashion, one can see that $\lambda$ is a convex function of temperature, becoming zero at $T=T_{s}$ [see Fig. 6(b)]. Thus, the splitting point is defined as a 


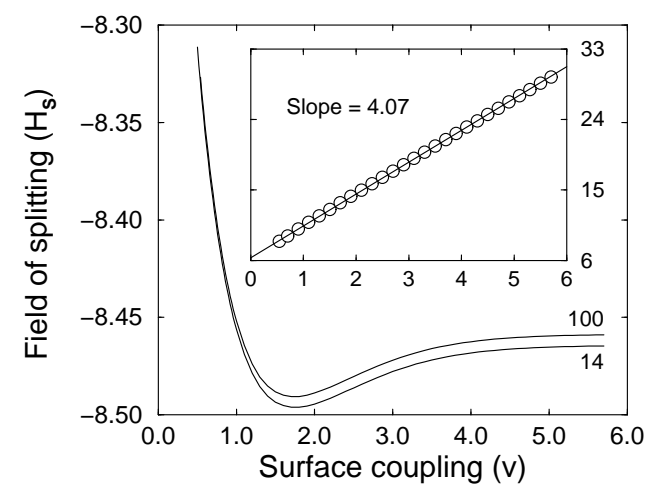

FIG. 7: Splitting field $H_{s}$ as a function of the surface coupling for AFM bcc(110) films with $N=14$ and $N=100$. The minimum of $H_{s}$ is due to the reentrance at low temperatures of the bulk critical curve. Inset: Splitting value of surface field $h_{s}$ as a function of the surface coupling $v$, for the case of $N=100$ (circles). A least-squares fit (solid line) gives $h_{s}=4.07 v+6.36$. Compare this with $h_{\mathrm{III}-\mathrm{VI}}=4 v+6$ obtained in Sec. II for the boundary between regions III and IV. See the text for further details.

saddle point of $\lambda$ in the $T$ and $H$ variables. Conditions $(10 \mathrm{~b})$ account for this.

Using conditions (10) we determined the splitting value of the external field $H_{s}$ as a function of the surface coupling for thin $(N=14)$ and thick $(N=100)$ films. The results are shown in Fig. 7 for the case of bcc(110) films. The particular shape of $H_{s}(v)$ can be understood as follows: Since the height of the critical curve associated with the surfaces scales with $v$ [see for example Figs. 5(b) and 6(a)] and because of the reentrance of the bulk critical curve, for small $v$ the point of contact (splitting) between the two critical curves is shifted to higher values of $H$. As we increase the surface coupling, the splitting point moves (clockwise) along the bulk critical curve, reaching a minimum in $H$ and increasing again towards the saturation value.

We found that within the PA the minimum in $H_{s}(v)$ is not very sensitive to the total number of layers. For $\operatorname{bcc}(110), H_{s}^{\min }$ occurs at $v \sim 1.74$ while for sc(100) the $H_{s}$ is minimum at $v \sim 1.2$. Again, this can be explained by considering the different Néel temperature values for sc and bcc lattices. The ratio between the latter and the former is $\sim 1.4$ (PA), which is comparable to the ratio of the corresponding $H_{s}^{\min }(\sim 1.45)$. The behavior of the other quantities of interest can be inferred from Fig. 7 . The most interesting part, however, is contained in the inset of Fig. 77, which shows $h_{s}$ as a function of the surface coupling $v$. A least-square fit gives $h_{s}^{\text {bcc }}=4.07 v+6.36$ which is almost parallel (and very close) to $h_{\text {III-IV in Eq. }}$ (8). For sc(100) films similar results were obtained and a linear fit for $h_{s}$ gives $h_{s}^{\mathrm{sc}}=4.04 v+5.20$. Thus, the process of splitting occurs within a narrow interval of $h$.

Due to the equivalence between the Néel point and the critical point of a ferromagnet in zero field, the finitetemperature behavior of AFM thin films, as a function

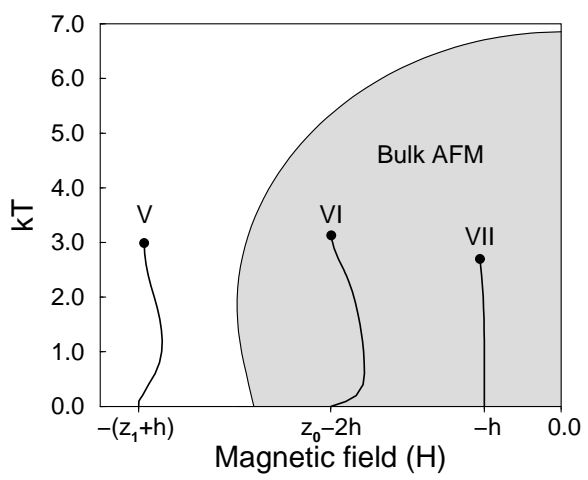

FIG. 8: A line of first-order transitions (thick line) appears in each of the regions $\mathrm{V}-\mathrm{VI}$ for negative values of the surface coupling. In all cases, the coexistence is between up- and down-ferromagnetism at the surfaces. In all cases the bulk critical curve is not affected by the presence of the first-order transitions. Since the antiferromagnetic domain (shaded region) is symmetric around $H=0$ only the left half is shown. The calculations were done in the PA-CVM for the following values of the surface variables: $v=-1$ and $h=2$ (VII); $h=5$ $(\mathrm{VI}) ; h=9(\mathrm{~V})$.

of the surface coupling $v$ and $H=h=0$, is equivalent to the multicritical phenomena occurring at the surface of semiinfinite ferromagnets. 49 In our case, negative surface pair interactions give rise to a line of first-order transitions in regions V-VII (see Fig. 8). In all cases the coexistence line separates surface ferromagnetic phases with opposite magnetization, that have the same symmetry. The bulk, however, may have different symmetry at each side of the coexistence line, thus modifying the shape of the first-order line at finite temperatures. This can be observed in Fig. 8, where the surface coexistence curve is drawn $v=-1$ and $h=2(\mathrm{VII}), h=5(\mathrm{VI}), h=9$ $(\mathrm{V})$. In each case, the coexistence curve ends in a critical point which is close, as expected, to the Curie point associated with the (2D) surface lattice, i.e. $\sim|v| T_{c}$. In all the three regions V-VII, the AFM bulk remains undisturbed by the presence of the surface coexistence line. At $v=v_{N}$ and $H=h=0$ the critical end point reaches the second-order critical curve at the Néel temperature $T=T_{N}$. The multicritical behavior is the (trivial) superposition of two independent fritical behaviors which do not interfere with each other.29

\section{SUMMARY AND CONCLUSIONS}

In this paper we performed an analysis of the confinement effects on antiferromagnets with symmetrypreserving surface orientations. The ground-state properties of the model, an Ising Hamiltonian with nn-pair interactions in the presence of external bulk and surface fields, shows an interesting structure. A zerotemperature phase diagram in the surface variables $v$ (surface coupling) and $h$ (surface field) was obtained for 
two-sublattice antiferromagnets. In this case there are seven different regions in the ground-state phase diagram. Each region is characterized by a particular sequence of ground states as a function of the external field. An analysis of the ground-state phase diagram explains (and sometimes even anticipates) some of the features found in the $H$-T critical curves. Together with an examination of the finite-temperature behavior in each of the aforementioned regions, our analysis showed that the interplay between the surface variables $v$ and $h$ defines the thermodynamics of confinement in ordering systems. For example, the splitting of the critical curve into surface and bulk contributions results from the simultaneous application of seemingly competing contributions $v>v_{m}$ (ordering) and $h>h_{\mathrm{II}-\mathrm{IV}}$. At the other extreme, the development of a surface coexistence line for $v<0$ and $h>0$ represents a particular case of magnetic surface reconstruction.

\section{Acknowledgments}

A.D.-O gratefully acknowledges the financial support from CONACyT through the Post Doctoral Fellowships Program and under grant G-25851-E.
1 M. E. Fisher and H. Nakanishi, J. Chem. Phys. 75, 5857 (1981).

2 H. Nakanishi and M. E. Fisher, J. Chem. Phys. 78, 3279 (1983).

3 R. Evans, J. Phys.: Condens. Matter. 2, 8989 (1990).

${ }^{4}$ K. Binder and D. P. Landau, J. Chem. Phys. 96, 1444 (1992).

5 R. Evans and U. M. B. Marconi, J. Chem. Phys. 86, 7138 (1987).

6 A recent review of phase transitions in polymer blends and block polymers in thin film geometries can be found in: K. Binder, Adv. Polym. Sci. 138, 1 (1999).

7 F. Brochard-Wyard and P. G. de Gennes, Compt. Rend. Acad. Sci. (Paris) Ser. II, 297, 223 (1983).

8 A. O. Parry and R. Evans, Phys. Rev. Lett. 64, 439 (1990).

9 A. O. Parry and R. Evans, Physica (Amsterdam) 181A, 250 (1992).

10 M. R. Swift, A. L. Owczarek, and J. O. Indekeu, Europhys. Lett. 14, 475 (1991).

11 K. Binder, D. P. Landau, A. M. Ferrenberg, Phys. Rev. Lett. 74, 298 (1995).

12 K. Binder, D. P. Landau, A. M. Ferrenberg, Phys. Rev. E 51, 2823 (1995).

13 K. Binder, R. Evans, D. P. Landau, and A. M. Ferrenberg, Phys. Rev. E 53, 5023 (1996).

14 A. M. Ferrenberg, D. P. Landau, and K. Binder, Phys. Rev. E 58, 3353 (1998).

15 A. Maciołek and J. Stecki, Phys. Rev. B 54, 1128 (1996).

16 J. Rogiers and J. O. Indekeu, Europhys. Lett. 24, 21 (1993).

17 E. Carlon and A. Drzewiński, Phys. Rev. Lett. 79, 1591 (1997).

18 E. Carlon and A. Drzewiński, Phys. Rev. E 57, 2626 (1998).

19 For reviews on wetting see: S. Dietrich, in: Phase Transitions and Critical Phenomena, edited by C. Domb and J. Lebowitz (Academic Press, New York, 1988), Vol. 12; D. E. Sullivan and M. M. Telo da Gama, in: Fluid Interfacial Phenomena, edited by C. A. Croxton (Wiley, New York, 1986); M. Schick, in: Liquids at Interfaces, edited by J. Charvolin, J. F. Joanny, and J. Zinn-Justin (NorthHolland, Amsterdam, 1990).

20 J. L. Morán-López, F. Mejía-Lira, and K. H. Bennemann, Phys. Rev. Lett. 54, 1936 (1985).

21 J. M. Sanchez and J. L. Morán-López, Phys. Rev. B 32,
3534 (1985).

22 F. Mejía-Lira, K. H. Bennemann, and J. L. Morán-López, Phys. Rev. B 32, 5925 (1985).

${ }^{23}$ For a recent review of Monte Carlo studies on surfaceinduced order see: K. Binder, in Cohesion and Structure of Surfaces (North-Holland, Amsterdam, 1995). Also see the contributions of K. Binder, D. P. Landau, F. Schmid, and W. Schweika in: Stability of Materials, edited by A. Gonis, P. E. A. Turchi, and J. Kudrnovský. NATO ASI Series B, Vol. 355 (Plenum, New York, 1996).

${ }^{24}$ For an experimental review on surface critical phenomena see: H. Dosch, Critical Phenomena at Surfaces and Interfaces, Springer Tracts in Modern Physics, Vol. 126 (Springer-Verlag, Berlin, 1992).

25 R. Lipowsky, Phys. Rev. Lett. 49, 1575 (1982).

26 R. Lipowsky, Ferroelectrics 73, 69 (1987).

27 C. Ebner, C. Rottman, and M. Wortis, Phys. Rev. B 28, 4186 (1983).

28 S. J. Kennedy and J. S. Walker, Phys. Rev. B 30, 1498 (1984). Binder and Landau (Ref. 29) performed a Monte Carlo investigation in the same model but in the context of magnetic surface reconstruction.

${ }^{29}$ K. Binder and D. P. Landau, Surf. Sci. 151, 409 (1985).

30 F. Schmid, Z. Phys. B 91, 77 (1993).

31 A. Drewitz, R. Leidl, T. W. Burkhardt, and H. W. Diehl, Phys. Rev. Lett. 78, 1090 (1997).

32 R. Leidl and H. W. Diehl, Phys. Rev. B 57, 1908 (1998).

33 S. Krimmel et al., Phys. Rev. Lett. 78, 3880 (1997).

34 U. Ritschel and P. Czerner, Phys. Rev. Lett. 77, 3645 (1996).

35 U. Ritschel, Phys. Rev. B 57, 693 (1998).

36 A. Díaz-Ortiz, J. M. Sanchez, and J. L. Morán-López, Phys. Rev. Lett. 81, 1146 (1998).

37 R. Kikuchi, Phys. Rev. 81, 998 (1951).

38 We confirmed these results by evaluating the free energy (in the pair approximation) at low temperatures, as function of the surface variables $h$ and $v$ for different values of the external field $H$. We did not find any other groundstate structure additional to those listed in Table 1 .

39 E. M. Sosa-Hernández, F. Aguilera-Granja, and J. L. Morán-López, Phys. Rev. B 52, 5392 (1995).

40 A. Díaz-Ortiz, J. M. Sanchez, and J. L. Morán-López, Compt. Mater. Sci. 8, 79 (1997).

41 A. Díaz-Ortiz, J. M. Sanchez, F. Aguilera-Granja, and J. L. Morán-López, Solid State Commun. 107, 285 (1998). 
${ }^{42}$ F. Ducastelle, Order and Phase Stability of Alloys (NorthHolland, Amsterdam, 1991).

43 D. de Fontaine, Solid State Phys. 34, 73 (1979); ibid. 47, 33 (1994).

44 A. Finel, in Statics and Dynamics of Alloy Phase Transformations, edited by P. E. A. Turchi and A. Gonis (Plenum, New York, 1994).

45 Foundations and Applications of the Cluster Variation and Path Probability Method, edited by T. Morita, M. Suzuki, K. Wada, and M. Kaburagi, Prog. Theor. Phys. Suppl. No. 155 (1994).

46 Theory and Applications of the Cluster Variation and Path Probability Methods, edited by J. L. Morán-López and J. M. Sanchez (Plenum, New York, 1996).
47 At finite temperatures, the antiferromagnetic region [nonzero value of the order parameter in (9)] is separated from the disordered region (zero long-range order) by a line of second-order phase transitions. Equation (10a) defines the loci of the transition points in terms of a second derivative of free energy of the system with respect of the order parameters.

48 J. M. Sanchez and D. de Fontaine, Phys. Rev. B 17, 2926 (1978).

49 For a review of the critical behavior at surfaces see: K. Binder, in Phase Transitions and Critical Phenomena, edited by C. Domb and J. L. Lebowitz (Academic, New York, 1983). 